\title{
Strengthening the Reporting of Observational Studies in Epidemiology (STROBE) in the International Journal of Medical Students
}

Aisha Gharaibeh, ${ }^{1}$ Sahil Koppikar, ${ }^{2}$ Francisco J. Bonilla-Escobar. ${ }^{3}$

\begin{abstract}
Observational studies are the foundation of all biomedical and clinical research.' While clinical trials and meta-analyses offer the highest levels of evidence and are therefore considered the gold standards of human research, experimental studies would be hardly possible without any evidence from the hypothesis-generating observational studies. It is by the help of weII-conducted epidemiological studies that scientists can inform public health decisions and clinical practice guidelines.
\end{abstract}

Nevertheless, observational epidemiological studies are not always conducted by expert epidemiologists. In such cases, fundamental deficiencies in reporting have been identified. ${ }^{2}$ Conflicting results from epidemiological studies on the risks of common daily products, such as the use of coffee, hair dyes, or hormones, are frequently and eagerly reported in the popular press, providing a constant source of anxiety and confusion to the public. This raises the need for guidelines on reporting such studies as medical journal editors might face problems upon reviewing these papers.

In the last few years, the STROBE checklist has been used frequently to analyze the reporting quality of published studies. ${ }^{3}$ This ensured that each section of the paper included the relevant items that would allow other researchers to reproduce, compare and analyze the information presented, such as the background and objectives in the Introduction section; study and sampling design, setting, participants, variables, data sources, bias, study size, quantitative variables and statistical analysis in the Methods section; participants and descriptive information, outcome data, main results and additional analysis in the Results section; and, finally, key results, limitations and generalizability in the Discussion section.

In September 2004, a meeting was held in the United Kingdom to identify and create common guidelines that could be used internationally for publication of observational studies. Experts in various fields attended this meeting, including epidemiologists, methodologists, statisticians, and editorial staff from the Annals of Internal Medicine, British Medical Journal (BMJ), Bulletin of the World Health Organization, International Journal of Epidemiology and the Journal of the American Medical Association (JAMA). They identified the most important items to be included in a checklist for each type of study and the strategy for disseminating this information, referred to as the STROBE (STrengthening the Reporting of OBservational studies in Epide- miology) Statement. In October 2007, the STROBE statement, a 22 -item checklist, was published in a number of journals. These items should be addressed in all articles that are reporting cross-sectional, case-control or cohort studies. The aim of the statement is to provide guidance on reporting observational studies in a consistent and thorough manner that can be easily reproduced.'

While clarity of reporting is a prerequisite to evaluation, the checklist should not be regarded as an instrument to evaluate the quality of observational research. Good reporting does not necessarily translate into quality research. The importance of good reporting is that others-readers, fellow scientists, reviewers and editors-can form an informed opinion on whether the research was appropriate and what aspects might need more scrutiny.

Since its inception, additional efforts have been taken by the STROBE Group to promote the STROBE guidelines by creating a number of extensions or additional statements similar to the first set of guidelines, such as:

- Strengthening the Reporting of Molecular Epidemiology for Infectious Diseases (STROME-ID). ${ }^{4}$

- REporting of studies Conducted using observational Routinely collected Data (RECORD). ${ }^{5}$

- STrengthening the Reporting of OBservational studies in Epidemiology: Molecular Epidemiology (STROBE-ME). It used 9 existing items of the STROBE Statement and provides $17 \mathrm{ad}$ ditional items related to molecular epidemiology. ${ }^{6}$

- Draft STROBE checklist for abstracts published on Sep 20th 2011. It has been tested at the European Congress of Clinical Microbiology and Infectious Diseases (ECCMID). The International Journal of Medical Students (IJMS) in the second Editorial of this issue is promoting its use.?

- Strengthening the reporting of genetic risk prediction studies: the GRIPS Statement. It was developed on the basis of other guidelines, such as STREGA, REMARK and STARD. The GRIPS statement consists of a checklist of 25 items to improve quality and transparency of the reporting of genetic risk prediction studies. ${ }^{8}$

The STROBE statement is referred to in the Uniform Requirements for Manuscripts Submitted to Biomedical Journals by the International Committee of Medical Journal Editors (ICMJE) (Available from: http://www.icmje.org/icmje-recommendations.

Scientific Editor International Journal of Medical Students (IJMS). Jordan University of Science and Technology, Ar Ramtha, Irbid, Jordan.

${ }^{2}$ Associate Editor of Short Communications IJMS. Queen's University, Kingston, Ontario, Canada.

${ }^{3}$ Editor in Chief IJMS. Cisalva Institute, School of Public Health, University of Valle, Cali, Colombia.

\section{Correspondence:}

Aisha Gharaibeh

Address: Jordan University of Science and Technology, Ar Ramtha, Irbid 22110, Jordan

Email: scientific.editor@ijms.info 


\section{Editorial}

pdf, updated 2013 Dec; cited 2014 Jun 22). We, in the IJMS, refer to the STROBE Statement in the Authors Guidelines (Available from: http://www.ijms.info/ojs/index.php/IJMS/about/submissions\#authorGuidelines, updated 2014 Jan 4; cited 2014 Jun 22). The IJMS Editorial Team is aware of this statement and the authors are requested to always follow the guidelines to improve the way that observational studies are reported. Furthermore, this helps achieve one of the main objectives of the IJMS, that is, to teach young scientists how to conduct transparent studies and to promote ethical and professional research among young professionals for scientific advancement.

\section{References}

1. von Elm E, Altman DG, Egger M, Pocock SJ, G $\emptyset$ tzsche PC, Vandenbroucke JP; STROBE Initiative. The Strengthening of the Reporting of Observational Studies in Epidemiology (STROBE) statement: guidelines for reporting observational studies. PLOS Med. 2007 Oct 16;4(10):e296.

2. Altman DG, De Stavola BL, Love SB, Stepniewska KA. Review of Survival Analyses Published in Cancer Journals. Br J Cancer. 1995 Aug;72(2):511-18.

3. Fung AE, Palanki R, Bakri SJ, Depperschmidt E, Gibson A. Applying the CONSORT and STROBE Statements to Evaluate the Reporting Quality of Neovascular Age-related Macular Degeneration Studies. Ophthalmology. 2009 Feb; 116(2):286-96.

4. Field N, Cohen T, Struelens MJ, Palm D, Cookson B, Clynn R, et al. Strengthening the Reporting of Molecular Epidemiology for Infectious Diseases (STROME-ID): an extension of the STROBE statement. Lancet Infect Dis. 2014 Apr;14(4):341-52.

5. Liyanage H, Liaw ST, de Lusignan S. Reporting of Studies Conducted using Observational Routinely Collected Data (RECORD) Statement: call for contributions from the clinical informatics community. Inform Prim Care. 2012;20(4):221-4.

6. Gallo V, Egger M, McCormack V, Farmer PB, loannidis JP, Kisch-Volders M, et al. STrengthening the Reporting of OBservational Studies in Epidemiology-Molecular Epidemiology (STROBE-ME). An Extension of the STROBE Statement. PLoS Med. 2011 Oct;8(10):e1001117.

7. Abhishekh HA, Tandon N, Rangarajan S, Gharaibeh A, Bonilla-Escobar FJ. Poster Presentations: A Presenting Opportunity in Conferences for Medical Students. Int J Med Students. 2014 Mar-Jun;2(2):38-9.

8. Janssens AC, Ioannidis JP, van Dujin CM, Little J, Khoury MJ; GRIPS Group. Strengthening the reporting of Genetic RIsk Prediction Studies: the GRIPS Statement. PLoS Med. 2011 Mar;8(3):e1000420.

\section{Acknowledgments}

None.

Conflict of Interest Statement a Funding

The Authors have no funding, financial relationships or conflicts of interest to disclose.

Cite as:

Gharaibeh A, Koppikar S, Bonilla-Escobar FJ. Strengthening the Reporting of Observational Studies in Epidemiology (STROBE) in the International Journal of Medical Students. Int J Med Students. 2014 Mar-Jun;2(2):36-7. 